\title{
Laryngeal ultrasound: a useful method in predicting post-extubation stridor. A pilot
} study

\author{
L-W. Ding**\#, H-C. Wang*, , H-D. Wu+, C-J. Chang ${ }^{\S}$ and P-C. Yang*
}

ABSTRACT: The cuff-leak test was widely used for the prediction of post-extubation stridor, but controversial results limit its clinical application. The current study used real-time ultrasonography to evaluate the air-leak and hypothesised that the air-column width, measured by ultrasonography, may be correlated to the development of post-extubation stridor.

From June 1, 2001 to March 1, 2002, a total of 51 planned extubations in 51 consecutively intubated patients were included. All of the patients received ultrasonographical examinations of their vocal cords and larynx in addition to an air-column width measurement within $24 \mathrm{~h}$ prior to extubation.

The overall post-extubation stridor rate was $7.8 \%$. The air-leak volume presented as median (interquartile range) were $300(350) \mathrm{mL}$ and $25(20) \mathrm{mL}$, respectively, for the nonstridor and stridor groups. The air-column width during cuff deflation was $6.4(2) \mathrm{mm}$ and $4.5(0.8) \mathrm{mm}$, respectively. They were found to be statistically significant.

In conclusion, the authors demonstrated that laryngeal ultrasonography could be a reliable, noninvasive method, in the evaluation of vocal cords, laryngeal morphology and the ease of airflow, which passed through vocal cords or subglottic area due to laryngeal oedema. The aircolumn width during cuff deflation was a potential predictor of post-extubation stridor.

\section{KEYWORDS: Cuff-leak test, laryngeal ultrasonography, post-extubation stridor}

E ndotracheal intubation may generate local complications, including mechanical lesions, such as friction and compressions between the tube and the anatomic structures [1] Laryngeal oedema occurs in $2-15 \%$ of patients after extubation [2, 3] and manifests as respiratory distress and inspiratory whistling called "stridor". Stridor in extubated patients involves a degree of morbidity and mortality $[2,4]$. There is no good method to identify patients at risk of post-extubation stridor and laryngeal oedema.

A reduced cuff-leak volume identifies a population at increased risk for the development of stridor after extubation. MiLler and COLE [5] reported that the cuff-leak test might be a useful index of clinically significant laryngo-tracheal narrowing. The cuff-leak test was also widely used in the evaluation of upper-airway patency, prior to extubation. However, ENGOREN [6] reported a high negative predictive value, but a low positive predictive value, for the cuff-leak test. Although the cuff-leak test is safe and simple, the controversial results may cause physicians to make difficult decisions regarding extubation if the cuff-leak test is positive.
Examination of the vocal cords (VC) is most commonly performed by direct or indirect laryngoscopy, but this may not be readily approached by some patients, especially infants, children and intubated patients. Post-extubation stridor is commonly the result of oedema of the subglottic area or the VC [7]. The difficulty in defining the relationship between laryngotracheal injury and post-extubation stridor is that the presence of the endotracheal tube precludes direct visualisation of the upper airway, prior to extubation. Other noninvasive approaches to examine the VC and the larynx would be helpful and there have been many ultrasonographical methods developed for visualising these [8-14]. Moreover, the current authors found that the motion of the VC, caused by air passage during respiration, could also be demonstrated using ultrasound (US).

Therefore, this prospective study was carried out to evaluate the morphology of the VC and the larynx by real-time, high-resolution US and to correlate the ultrasonographical features with the images detected by fiberoptic bronchoscopy.

\section{AFFILIATIONS}

*Dept of Internal Medicine, +Division of Respiratory Therapy, Dept of Integrated Diagnotherapeutics,

${ }^{\text {s}}$ Dept of Medical Research, National Taiwan University Hospital, and -Dept of Internal Medicine, Far Eastern Memorial Hospital, Taipei, and

\#Unit of Critical Care Medicine, Lotung Poh-Ai Hospital, Yi-Lan, Taiwan.

CORRESPONDENCE

$\mathrm{H}-\mathrm{C}$. Wang

Dept of Internal Medicine

National Taiwan University Hospital

No. 7

Chung-Shan South Road

Taipei 100

Taiwan

Fax: 886223582867

E-mail: hcwang@ha.mc.ntu.edu.tw

Received:

March 142005

Accepted after revision:

September 122005

SUPPORT STATEMENT

This study was equally supported by funds from Hao-Chien Wang and Chee-Jen Chang. 


\section{MATERIALS AND METHODS Patients}

A total of 51 consecutive intubations, on 51 different mechanically ventilated adult patients from June 1, 2001 to March 1, 2002 were studied. All of the patients were admitted to the medical intensive care unit (ICU) of the National Taiwan University Hospital (Taipei, Taiwan). The inclusion criteria for the subjects were admission to the medical ICU and intubation for a minimum of $24 \mathrm{~h}$ for acute respiratory failure, other various causes, or for airway protection. All of the patients were intubated with a soft, high-volume, low-pressure cuffed endotracheal tube (ETT) with an internal diameter size of 6.5$8 \mathrm{~mm}$. A mechanical ventilator (Nellcor-Puritan-Bennett 7200; Nellcor-Puritan-Bennett Inc, California, CA, USA) was also used. The patients were sedated on assist-control ventilatory support to ensure passive inspiration and expiration during the cuff-leak test and laryngeal US. The ethics committee of the hospital approved the study and informed consent was obtained from all of these patients.

\section{Protocol}

Within $24 \mathrm{~h}$ prior to the planned extubation, which was decided by the clinical physicians, the cuff-leak test, laryngeal US and bronchoscopy were performed. The cuff-leak test was performed as described by MILLER and COLE [5]. In brief, the respiratory therapist placed the ventilator in assist-control mode with $5 \mathrm{cmH}_{2} \mathrm{O}$ of positive end-expiratory pressure and adjusted the tidal volume $(V \mathrm{~T})$ until the peak airway pressure was up to $30 \mathrm{cmH}_{2} \mathrm{O}$. The patients' endotracheal and oral secretions were gently suctioned, and then the endotracheal tube cuff was deflated. The set inspiratory $V \mathrm{~T}$, peak airway pressure and the six subsequent exhaled volumes were recorded. The lowest three values were averaged.

The cuff-leak volume was defined as the difference between the preset inspiratory $V \mathrm{~T}$ and the average of the three lowest exhaled volumes. The physicians decided the mode of weaning and the timing of extubation based on their assessment of gas exchange and respiratory reserve. Each patient was demonstrated to have an ability to tolerate $\geqslant 30 \mathrm{~min}$ of spontaneous respiration with a T-piece on the day of extubation. All of the patients were extubated within $4 \mathrm{~h}$ after both the cuff-leak test and laryngeal US had been taken. The results were not made available to the physicians and the patients were extubated regardless of the results.

The laryngeal US was performed with an Aloka SSD-1400 US machine equipped with a $5.0 \mathrm{MHz}$ probe for the visualisation of the VC (ALOKA Co., LTD., Tokyo, Japan). The investigators performing the US did not know the results of the cuff-leak tests. The patients were in the supine position, with the neck hyper-extended. The probe was placed on the cricothyroid membrane with a transverse view of the larynx (fig. 1). To avoid the examination bias and artefacts created by different investigators, the standard scanning plane was predetermined: it should contain several landmarks, including the VC, false cords, thyroid cartilage and arytenoids cartilage as demonstrated in figures $1 b, 2 a$ and $2 b$. This sonographical technique could make a comparable and complete study possible through the unanimous anatomical-section plane by different investigators. It was performed with the balloon cuff inflated, after the oral and laryngeal secretions were suctioned and all of

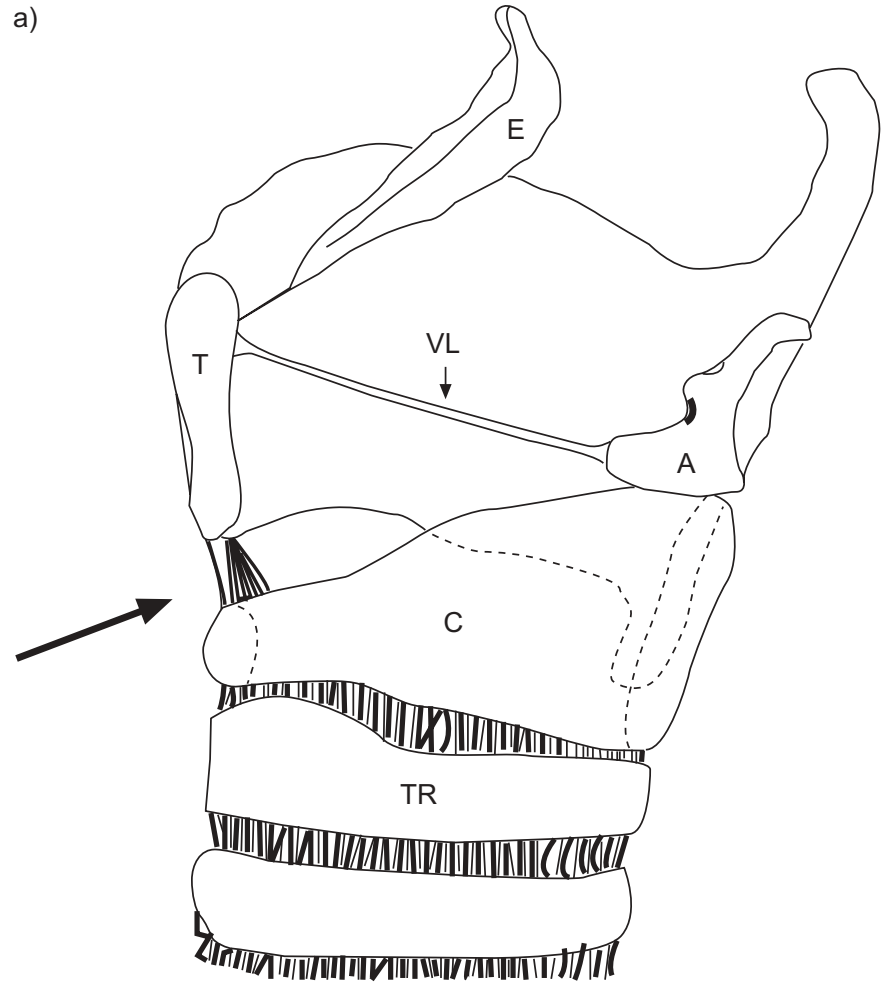

b)

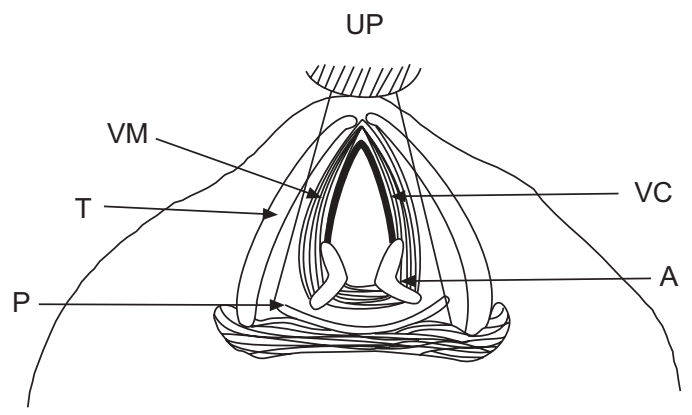

FIGURE 1. a) Transaxial views of the vocal cords (VC). The arrow indicates the direction of the scanning plane. b) Axial section through the larynx shows the position of the ultrasound probe (UP) and the VC. A: arytenoid cartilage; C: cricoid cartilage; E: epiglottis; $P$ : pharynx; $T$ : thyroid cartilage; TR: tracheal ring; VL: vocal ligament; VM: vocalis muscle.

the settings were the same as the cuff-leak test. The laryngeal US was repeated with the balloon cuff deflated.

The vocal cords, surrounding soft tissues and the air passage through the vocal cords were observed. The laryngeal aircolumn width was defined as the width of air passed through the VC as determined by US (fig. 2). The air-column difference was the width difference between balloon-cuff inflation (BI; fig. $2 \mathrm{a}$ and $2 \mathrm{~b}$ ) and balloon-cuff deflation (BD; fig 2c and 2d). Two different physicians recorded the air-column width during $\mathrm{BD}$ and $\mathrm{BI}$ over the respiratory cycles for three consecutive times, the averaged value was recorded. Bronchoscopic examination was performed in all patients under sedation, sequentially after the cuff-leak test and laryngeal US were performed prior to extubation. 

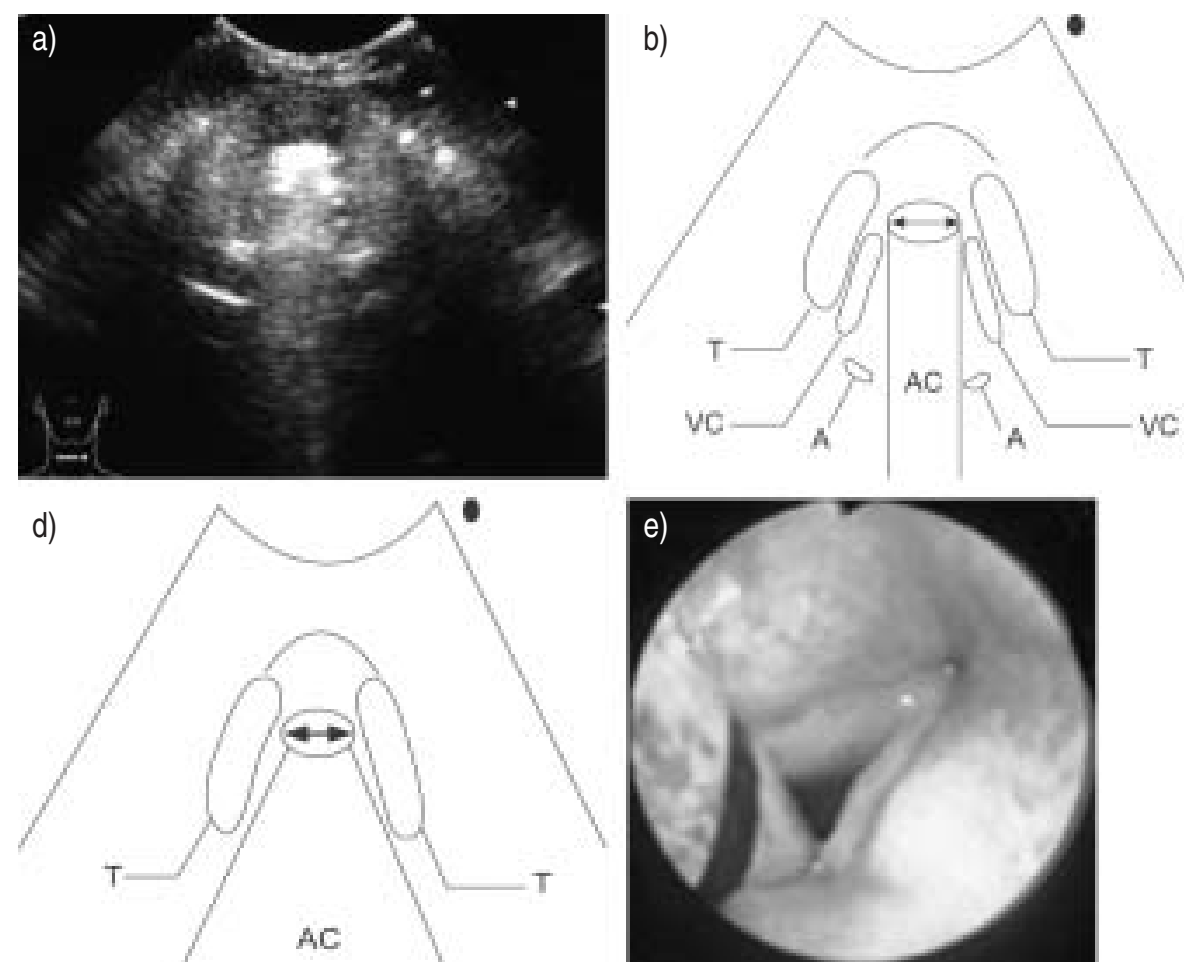

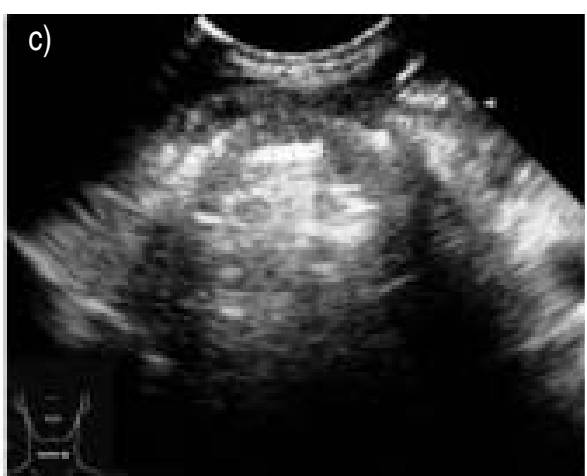

FIGURE 2. a) A typical ultrasound (US) image of the laryngeal air-column during ballon-cuff inflation. Square-shaped air-column with hyper-echoic air-column bands and acoustic shadows are shown. Paired hypoechoic true cords are over both sides of the air-column. Hyper-echoic arytenoid cartilages are behind the true vocal cords and beside the air-column. b) Drawing of a). c) Typical US image of the laryngeal air-column during balloon-cuff-deflation (BD) in the same patient as a), who did not develop post-extubation stridor. The air-column became trapezoid in shape and the air-column width increased. The arytenoid cartilage and part of the true cords were masked by the acoustic shadow. d) Drawing of c). e) The bronchoscopic image of the vocal cords during $\mathrm{BD}$ in the same patient as a). A arytenoids cartilage; $\mathrm{AC}$ : laryngeal air-column; $\mathrm{T}$; thyroid cartilage; VC: true vocal cords. Arrow shows air-column width.
Stridor was defined as the presence of a high-pitched inspiratory wheeze localised to the trachea or the larynx and was associated with respiratory distress, usually requiring medical intervention. All of the assessments for stridor, respiratory distress and the need for re-intubation were made by the ICU physicians who were blinded to the measurements obtained by the investigators. The management of stridor included epinephrine aerosol inhalation, bi-level positive airway pressure (BiPAP) support and either inhalation or intravenous steroids. Re-intubation due to post-extubation stridor was defined as the patient, after extubation, presenting with stridor, impending respiratory failure and symptoms of respiratory distress, all of which were immediately relieved after intubation.

\section{Statistical analysis}

Statistics for both basic demographics and all variables suspected to be correlated to post-extubation stridor were displayed by the stridor status. Descriptive statistics, such as median and interquartile values, were shown for all continuous variables, e.g. as age, intubated duration, cuff-leak volume, air-column difference, air-column width during balloon deflation and peak-airway-pressure difference between $\mathrm{BD}$ and $\mathrm{BI}$. Comparisons between nonstridor and stridor groups were made utilising both parametric and nonparametric approaches; namely, two-sample $t$ - and two-sample Wilcoxon-rank sum test for continuous variables; two-sample Chi-square and Fisher Exact tests for categorical variables. All statistical tests were two-sided and were evaluated at the 0.05 level of significance with $95 \%$ confidence intervals (CI) provided.

\section{RESULTS}

As patients who entered this study would have to undergo the cuff-leak test, laryngeal US and bronchoscopy, only 51 patients gave their informed consent. The demographic characteristics of these patients are shown in table 1.

\section{Ultrasonographical features of the vocal cords and larynx}

The VC were delineated, sonographically, in the transverse plane, through the anterior neck at the level of the cricothyroid membrane and the air-column was clearly demonstrated. The air-column was square-shaped and the air-column width could easily be measured. The arytenoid cartilage was clearly shown when the cuff balloon was inflated (fig. 2a and b). In contrast, the air-column became trapezoidal in shape and masked the arytenoid cartilage when the balloon cuff was deflated (fig $2 c$ and $d$ ). The width of the top of the trapezoidal air-column was measured. Hence, the air-column width difference could easily be measured. The false VC were also visualised, using US, as paired, hyperechoic structures, due to the presence of fibrofatty tissues. The true VC appeared as hypoechoic structures because they consisted of muscles.

\section{Correlation of laryngeal ultrasonography with fiberoptic bronchoscope images}

The laryngeal US had a very good correlation with bronchoscopic images. Typical US and bronchoscopic images were demonstrated (figs 2 and 3). The correlation between the US and bronchoscopy findings could be demonstrated in the figures. The landmarks seen by the laryngeal US, such as VC and false cords, could also be seen directly by bronchoscopy. The air passing through the VC, whether cuff inflation or deflation, could be shown by the change in the air-column width. This could be correlated with bronchoscopic images of oedematous VC adapted from BENJAMIN [15]. In a patient with post-extubation stridor, necessitating re-intubation, the bronchoscope examinations of the VC showed swelling, oedematous change (fig. 4) and little changes of space, 


\begin{tabular}{|c|c|c|c|}
\hline \multirow[t]{2}{*}{ TABLE 1} & \multirow[b]{2}{*}{ Nonstridor ${ }^{\#}$} & d variable & \multirow[b]{2}{*}{ p-value ${ }^{+}$} \\
\hline & & Stridor & \\
\hline Age yrs & $71(20)$ & $67(10.5)$ & 0.57 \\
\hline Intubated days & $6(7)$ & $7(6)$ & 0.99 \\
\hline Sex male n (\%) & $34(72.3)$ & $1(25)$ & $0.04^{\S}$ \\
\hline Cuff-leak volume $\mathrm{mL}$ & $300(350)$ & $25(20)$ & $<0.01^{\S}$ \\
\hline $\begin{array}{l}\text { Peak airway pressure } \\
\text { difference } \mathrm{cmH}_{2} \mathrm{O}\end{array}$ & $6(8)$ & $3.5(1.8)$ & 0.08 \\
\hline $\begin{array}{l}\text { Air-column width on } \\
\text { BD mm }\end{array}$ & $6.4(2)$ & $4.5(0.8)$ & $0.01^{\S}$ \\
\hline $\begin{array}{l}\text { Air-column } \\
\text { difference } \mathrm{mm}\end{array}$ & $1.5(1.3)$ & $0.35(0.5)$ & $<0.01^{\mathrm{s}}$ \\
\hline ETT size mm & $7.5(0)$ & $7.5(0.30)$ & 0.51 \\
\hline 6.5 n (\%) & $2(4.3)$ & $0(0)$ & \\
\hline 7.0 n (\%) & $5(10.6)$ & $1(25)$ & \\
\hline 7.5 n (\%) & $36(76.6)$ & $3(75)$ & \\
\hline 8.0 n (\%) & $4(8.5)$ & $0(0)$ & \\
\hline
\end{tabular}

Data presented as median (interquartile range) unless otherwise stated. BD: balloon-cuff deflation; ETT: endotracheal tube. ${ }^{*}: \mathrm{n}=47 ;{ }^{\bullet}: \mathrm{n}=4{ }^{+}{ }^{+}$: using Wilcoxon-rank sum test ; ${ }^{\text {s: }}$ statistically significant.

between the VC, resulted from the air passing through the VC during $\mathrm{BD}$. The US of the VC also revealed increased VC width and no change in the air-column width (fig. 4). However, increased air-column difference and air-leak volume were noted after 10 days and bronchoscope examinations also showed that the oedema of the VC had subsided and the space between the VC had increased.

\section{Air-column width during balloon-cuff deflation accurately predicts the occurrence of post-extubation stridor}

Post-extubation stridor occurred in $7.8 \%$ of the subjects studied and the overall re-intubation rate was $9.8 \%$ of all extubations. Comparisons between nonstridor and stridor groups were made. Age, duration of intubation, and ETT size were performed individually. All coefficients had p-values $>0.05$.
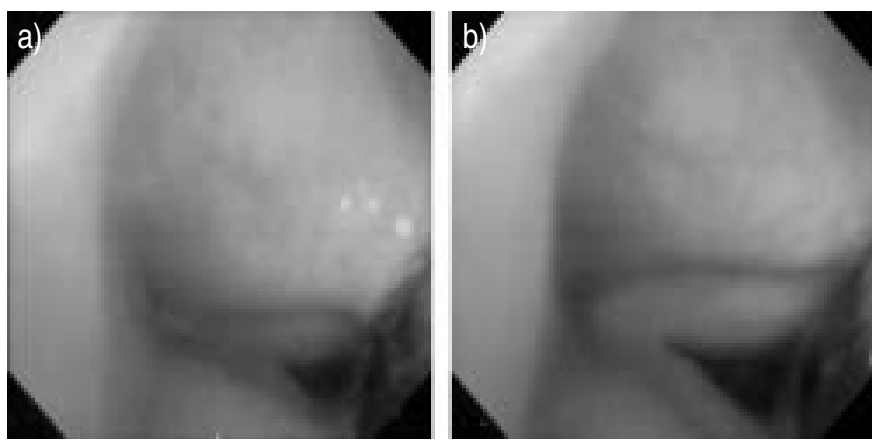

FIGURE 3. a) The bronchoscopic image of the vocal cords (VC) during balloon-cuff inflation $(\mathrm{BI})$ in a patient without post-extubation stridor. The motion of the VC and the space between them are limited during BI. b) The bronchoscopic image of VC during balloon-cuff deflation in the same patient as a); the distance between the VC is lengthened.
This led to a simplified model containing sex, cuff-leak volume, air-column difference, air-column width during balloon deflation and peak airway pressure difference, as the key variables for the prediction of post-extubation stridor (table 1).

The mean cuff-leak volume was significantly different between the stridor and the nonstridor groups. The mean air-column difference was significantly lower in those who developed stridor, when compared with those who did not. The aircolumn width during BD was significantly lower in patients who developed stridor (table 1).

Four patients presented with post-extubation stridor and they received aerosol epinephrine inhalation, steroid inhalation, or BiPAP support (table 2). The stridor of two patients subsided after this treatment the other two patients required reintubation, because the stridor was associated with respiratory failure. The stridor and symptoms of respiratory distress subsided immediately after re-intubation. One of these subjects was extubated successfully 10 days later without stridor.

\section{DISCUSSION}

Post-extubation stridor may occur in $2-15 \%$ of intubated patients. It can cause serious complications and even death, particularly in those patients that need re-intubation $[2,16]$. A
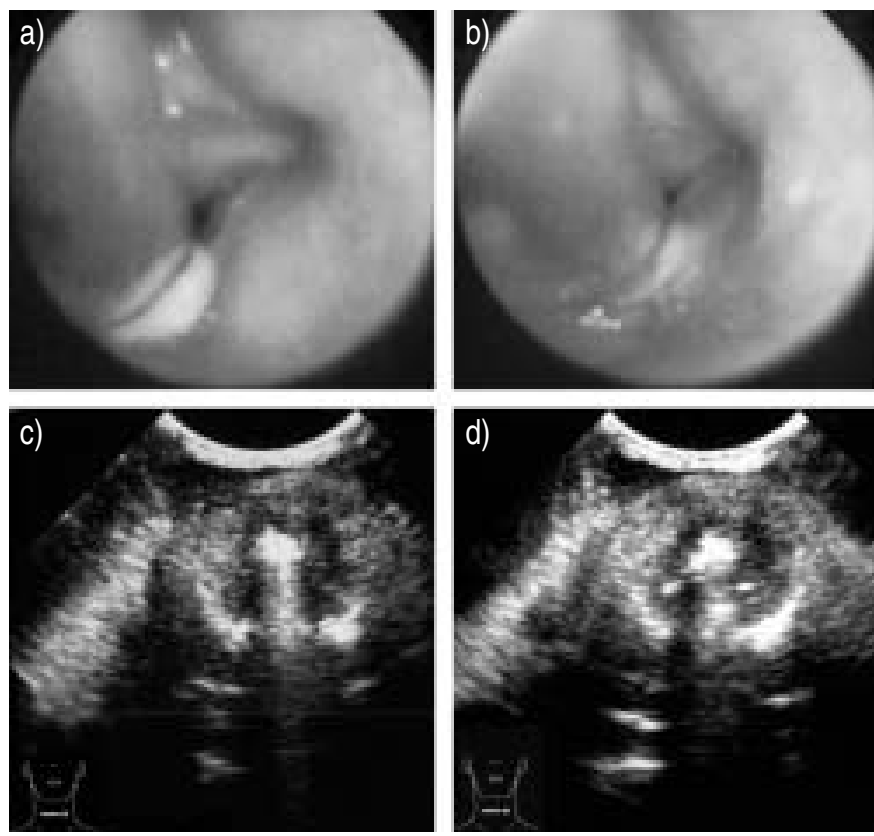

FIGURE 4. a) The bronchoscopic image of the vocal cords (VC) reveals the oedematous VC and the narrow space between them during balloon-cuff inflation (BI). b) The bronchoscopic image of the VC during balloon-cuff deflation (BD) in the same patient as part a). It reveals the oedematous $V C$ and the narrow space between $\mathrm{VC}$, even during $\mathrm{BD}$. Stridor developed in this patient after extubation and eventually re-intubation became necessary. c) The laryngeal ultrasound (US) of the same patient as part a) during Bl. Typical square-shaped air column with hyperechoic air-column bands and acoustic shadows are shown. d) The laryngeal US during $\mathrm{BD}$, in the same patient as part a), demonstrated no change in aircolumn width. The shape of the laryngeal air-column was as square as the image during $\mathrm{BI}$. The true cords and arytenoid cartilage were not masked by the aircolumn 
simple and safe test that can predict post-extubation stridor should become the standard method in the evaluation of upper-airway patency before extubation. Although the cuffleak test, as defined by MiLLER AND COLE [5] is safe and simple to perform, data from ENGOREN [6] showed great controversy in its accuracy and low positive predictive value $(0 \%)$ in the cardiac surgery population. Moreover, many factors may affect the cuff-leak volume. It may depend not only on the leak area, but also on the resistance in the endotracheal tube, the mechanical ventilator, and the exhaled limb of the breathing circuit. Tubes that are partially occluded by secretions may increase resistance to airflow and affect an increase in the cuffleak volume. The amount and method of applying positive pressure to the breathing circuit, the head and neck position and the presence or absence of sedation and neuromuscular blockade may all influence the cuff-leak test. Although the cuff-leak test is widely used in the evaluation of upper-airway patency, before extubation, its predictive value and accuracy is still unconvincing for ICU physicians.

Laryngeal US is a new, less invasive, and easily reproducible method of examining the larynx in infants and children [13]. This method is less invasive than endoscopy. It was demonstrated by the current authors that US can easily evaluate laryngeal morphology in intubated patients and that the laryngeal air column could be clearly seen, especially the dynamic changes during BI and deflation. The false VC, due to its increased echogenicity, is readily seen and easily differentiated from the true VC. In the current study, the serial changes of the VC and the air column were clearly shown through bronchoscopical and ultrasonographical images in a patient who developed post-extubation stridor. The BD or BI didn't alter the anatomy of the larynx. The change in the aircolumn width may reflect the change of air leak and airflow around the endotracheal tube. The air leak around the endotracheal tube is lower in those patients who developed stridor, the lower air column width could simply be the consequence of a lower airflow around the tube. Thus, the aircolumn width during BD measured by US has a potential ability to predict post-extubation stridor in intubated patients.

In clinical practice, physicians can easily evaluate the weaning potential in intubated patients, but it is difficult to predict the extubation failure due to upper-airway obstruction. Although ETTs with soft high-volume, low-pressure cuffs are used, laryngeal injury such as laryngeal granuloma, ulcer, necrosis and laryngeal oedema still occur. Pre-extubation evaluation of these patients can hardly be achieved because the ETT blocks direct visualisation of the larynx, trachea and VC. Although the cuff-leak test has been used for years, its accuracy and predictive value is still questionable.

In the present study, factors such as intubation duration, ETT size, and age did not differ significantly between patients with post-extubation stridor and those without. We found the aircolumn difference, air-column width during BD on laryngeal US correlated with post-extubation stridor. According to the current study's authors, the laryngeal US could be correlated well with the bronchoscopic findings of the motility and morphology of the VC. It could also evaluate the motion of the VC during BD. The laryngeal air column represented the air passing through the VC. With the balloon cuff deflated, the air passes by a peritubular space and through the VC. If there was a subglottic oedema or vocal cord oedema, air could hardly pass out.

The air-column width could be quantified by US and might represent the space between VC during BI and BD. The aircolumn difference represented the motility of the VC and the amount of air that passed through the VC between BI and BD. If airway obstruction resulted from subglottic oedema or VC oedema or paralysis, the air-column difference and air-column width during BD would be limited. The overall re-intubation rate was $9.8 \%$ while the rate of post-extubation stridor was $7.8 \%$, which is similar to previous reports $[2,16-17]$. In the current study only the air-column width, during BD was found to be a significant predictor for post-extubation stridor. The cuff-leak volume used traditionally to predict the postextubation stridor was not statistically significant. Because of the low incidence of post-extubation stridor, a larger patient group to achieve the optimal cuff-off point of air-column width during BD is needed.

However, the potential limitations of present study should be considered. First, airway secretions encrusted on the outer part of the endotracheal tube beneath the VC may affect the cuffleak volume and flow that may influence the air-column width and difference. The authors tried to reduce this phenomenon by gentle suction before the measurement. Second, the tracheal tube size may affect the air-column width. In the present study, the correct size of endotracheal tube was chosen, as in general practice. The size of endotracheal tube ranged from

TABLE 2 Medical characteristics of patients with post-extubation stridor

\begin{tabular}{lcccc} 
Patient & Medical history & Indication for intubation & Treatment for stridor & outcome \\
\hline $\mathbf{1}$ & CAD 3vd, HTN & Cardiogenic shock & Steroids, BiPAP support & Stable $^{\#}$ \\
$\mathbf{2}$ & COPD, CHF & Pneumonia & Epi, steroids, BiPAP support & Re-intubation", tracheostomy $^{\text {Re-intubation }^{+}}$ \\
$\mathbf{3}$ & Guillain-Barré syndrome & Pneumonia, respiratory muscle fatigue & Epi, steroids & Stable $^{\#}$ \\
$\mathbf{4}$ & DM, ESRD, HTN & UGl bleeding with hypovolaemic shock & Epi, BiPAP support & \\
\hline
\end{tabular}

CAD 3vd: coronary artery disease, with three vessels disease; HTN: hypertension; BiPAP: bi-level positive airway pressure; COPD: chronic obstructive pulmonary disease; CHF: congestive heart failure; Epi: racemic epinephrine nebulised; DM: diabetes mellitus; ESRD: end-stage renal disease. ${ }^{\#}$ : Symptoms subsided after

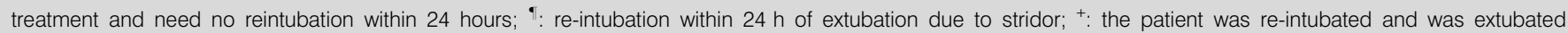
successfully, without stridor, 10 days later. 
6.5-8.0 $\mathrm{mm}$. The same patient may have quite different aircolumn width of air leak. It might be impossible to change the size of endotracheal tube and recheck the air-column width in the same patient. It is a potential limitation on the prediction capacity of both tests by the influence of tracheal tube size on the air-column width and cuff-leak volume. Third, cough could occur while the cuff balloon is deflating and the leak volume and the air-column width would increase. To reduce these influences and achieve the possible steady conditions, all of the patients were sedated during the measurement and the data were collected during the period without cough. The influence by cough could be avoided. However, the validity of the laryngeal US in awake patients, with or without cough, cannot be shown in this study. Fourth, the current study was conducted in a medical ICU, where the population (medical and surgical patients) factors should also be considered. Lastly, there is a difference in anatomical structure between the paediatric and adult airway. The paediatric airway is narrowest at the cricoid cartilage while the adult airway is narrowest at the larynx. Thus, the application of laryngeal US in paediatric patients needs further study.

Ultrasonography is a useful and non-invasive tool for the evaluation of vocal cords and laryngeal morphology in intubated patients. The air-column width measured by US may potentially identify patients at risk of post-extubation stridor, in whom caution should be taken after extubation. This study was a pilot study that suggested a potential diagnostic tool using air-column width during balloon deflation by laryngeal ultrasonography for prediction of post-extubation stridor other than cuff-leak test. Further studies, with larger patient numbers to overcome the low incidence of stridor, are needed to achieve an optimal cuff-off point for air-column width during balloon deflation.

\section{REFERENCES}

1 Steen JA, Lindholm CE, Brdlik GC, Foster CA. Tracheal tube forces on the posterior larynx: index of laryngeal loading. Crit Care Med 1982; 10: 186-189.

2 Rashkin MC, Davis T. Acute complication of endotracheal intubation. Chest 1986; 89: 165-167.

3 Colice GL, Stukel TA, Dain B. Laryngeal complication of prolonged intubation. Chest 1989; 96: 877-884.
4 Torres A, Gatell JM, Aznar E, et al. Re-intubation increases the risk of nosocomial pneumonia in patients needing mechanical ventilation. Am J Respir Crit Care Med 1995; 152: 137-141.

5 Miller RL, Cole RP. Association between reduced cuff leak volume and post-extubation stridor. Chest 1996; 110: 1035-1040.

6 Engoren M. Evaluation of the cuff-leak test in a cardiac surgery population. Chest 1999; 116: 1029-1031.

7 Benjamin B. Prolonged intubation injuries of the larynx: endoscopic diagnosis, classification, and treatment. Ann Otol Rhinol Laryngol 1993; 160: Suppl., 1-15.

8 Hertz CH, Lindstrom K, Sonesson B. Ultrasonic recording of the vibratory vocal cords. Acta Otolaryngol 1970; 69: 223.

9 Miles KA. Ultrasound demonstration of vocal cord movements. Br J Radiol 1989; 62: 871-872.

10 Raghavendra B, Horii S, Reede DL, Rumancik WM, Persky M, Bergeron T. Sonographic anatomy of the larynx, with particular reference to the vocal cords. J Ultrasound Med 1987; 6: 225-230.

11 Ueda D, Yano K, Okuno A. Ultrasonic imaging of the tongue, mouth, and vocal cords in normal children: establishment of basic scanning positions. J Clin Ultrasound 1993; 21: 431-439.

12 Carp H, Bundy A. A preliminary study of the ultrasound examination of the vocal cords and larynx. Anaesth Analg 1992; 75: 639-640.

13 Garel C, Contencin P, Polonovski JM, Hassan M, Narcy P. Laryngeal ultrasonography in infants and children: a new way of investigating normal and pathological findings. Int J Pediatr Otorhinolaryngol 1992; 23: 107-115.

14 Shih JY, Lee LN, Wu HD, et al. Sonographic imaging of the trachea. J Ultrasound Med 1997; 16: 783-790.

15 Benjamin B. Prolonged intubation injuries of the larynx: endoscopic diagnosis, classification, and treatment. Ann Otol Rhinol Laryngol 1993; 160: Suppl., 1-15.

16 Darmon JY, Rauss A, Dreyfuss D, et al. Evaluation of risk factors for laryngeal edema after tracheal extubation in adults and its prevention by dexamethasone. Anesthesiology 1992; 77: 245-251.

17 Epstein SK. Etiology of extubation failure and the predictive value of the rapid shallow breathing index. Am J Respir Crit Care Med 1995; 152: 545-549. 